\title{
EchoGéo
}

$23 \mid 2013$

Entre l'ancien et le nouveau monde. La transcendance du lieu et de l'espace en géographie vinicole contemporaine

\section{Le monde du vin}

Jean-Louis Chaléard

\section{OpenEdition}

\section{Journals}

Édition électronique

URL : https://journals.openedition.org/echogeo/13383

DOI : $10.4000 /$ echogeo.13383

ISSN : 1963-1197

Éditeur

Pôle de recherche pour l'organisation et la diffusion de l'information géographique (CNRS UMR 8586)

Référence électronique

Jean-Louis Chaléard, «Le monde du vin », EchoGéo [En ligne], 23 | 2013, mis en ligne le 05 juin 2013, consulté le 11 août 2021. URL : http://journals.openedition.org/echogeo/13383 ; DOI : https://doi.org/ 10.4000/echogeo. 13383

Ce document a été généré automatiquement le 11 août 2021.

EchoGéo est mis à disposition selon les termes de la licence Creative Commons Attribution - Pas d'Utilisation Commerciale - Pas de Modification 4.0 International (CC BY-NC-ND) 


\title{
Le monde du vin
}

\author{
Jean-Louis Chaléard
}

1 La nouvelle livraison d'Échogéo est largement consacrée à la vitiviniculture puisqu'elle fait l'objet du dossier de la rubrique Sur le champ et est illustrée par le dossier de la rubrique Sur l'image. Qu'une revue française à dominante géographique s'intéresse à la vigne et au vin n'a rien de surprenant. Au-delà des célèbres travaux de Roger Dion, de très nombreux géographes français ont travaillé ou travaillent sur la vigne, la viticulture et les pays de vignoble, en faisant un des sujets classiques de la discipline. Sans prendre le contre-pied de cette riche veine, le présent numéro d'ÉchoGéo a voulu aborder la question différemment, avec un dossier dirigé par notre collègue Glenn Banks, originaire d'un de ces nouveaux pays producteurs qui ont contribué à changer la géographie de la vigne dans le monde.

2 Cet éditorial aurait pu être intitulé Mondovino, du titre du célèbre documentaire de Jonathan Nossiter. Mais il ne s'agit ici ni de dénoncer ni d'attaquer violemment certains milieux liés à la vigne et au vin comme le fait le film. Sans compter que Mondovino est devenu aussi le nom d'un site de vente de vin en ligne sur internet... En revanche, c'est bien de vigne, de mondialisation et du monde qui tourne autour du vin qu'il s'agit.

3 Le dossier Sur le champ rassemble sept articles qui ne prétendent pas faire l'analyse exhaustive d'un sujet complexe, mais qui permettent de souligner quelques grandes évolutions. Sans reprendre les propos de Glenn Banks dans son introduction, on peut relever quelques points saillants. Ce qui frappe d'abord, à la lecture des articles, c'est la diversité des vignobles dans le monde, avec le maintien ou l'extension de la vigne là où on aurait pu la croire disparue (pensons au Royaume-Uni !), et son développement dans le domaine tropical (comme en Thaïlande). Si deux articles abordent la vigne en France, vieux pays de vignoble, une large place est faite aux nouveaux producteurs : Australie, Afrique du Sud, Nouvelle Zélande... L'expansion de la vigne dans le monde a conduit à une surproduction à laquelle chaque vignoble doit faire face, en fonction de son contexte particulier, et dont les cas étudiés ici fournissent plusieurs exemples. Par ailleurs, la mondialisation des échanges n'élimine pas les questions anciennes, mais amène à les renouveler, que ce soit le rôle des sols, du contexte économique ou surtout de la société et de la culture locales. Les questions de «terroirs» ou d'« Appellations 
d'Origine Contrôlée » sont intimement liées à celles relevant de la qualité, des goûts, mais aussi de la concurrence et de la protection de la production dans un monde d'échanges globalisés. De nouvelles questions émergent, avec l'évolution des sociétés, comme celle du vin "bio», ou avec les changements climatiques, qui ont des conséquences fortes dans les régions méditerranéennes.

4 Le dossier Sur l'image de Cécile Faliès et Julie Le Gall complète cette étude en l'illustrant par les cas de «nouvelles » régions productrices au Chili et en Argentine. Partant des images, il permet de s'interroger sur le rôle de la vigne dans la création des paysages ruraux à forte identité, dans des régions où l'arrivée de capitaux étrangers et la production de "vins de cépages» pourraient laisser penser a priori à l'absence d'identité locale. Il confirme la claire influence de la vigne sur les territoires au sein desquels elle se développe. Le cortège des cas étudiés dans cette livraison d'ÉchoGéo permet ainsi de dresser les contours d'une géographie mondialisée mais qui s'ancre fortement dans le local.

5 Certains lecteurs pourront s'étonner à propos du dossier sur le vin de la large place faite aux articles écrits en anglais. Est-il besoin de rappeler que la parution de ce numéro n'a rien à voir avec les débats qui ont agité en mai 2013 l'Assemblée nationale française et certains milieux intellectuel à propos de la place de l'anglais à l'université, ce numéro ayant été programmé depuis plusieurs mois. ÉchoGéo est une revue francophone et qui tient à le rester. Pour autant cela ne signifie pas un enfermement sur le champ clos de la francophonie. Nous avons déjà publié des articles en anglais, en espagnol aussi, et nous continuerons à le faire, dans le souci d'élargir notre public et partant, de faire connaître les travaux de la géographie française.

6 À côté du dossier sur le vin, les autres rubriques habituelles de la revue sont d'allure plus classique. La rubrique Sur le vif s'est enrichie d'un article sur le Mali. Ce ne sera pas le seul car les évènements qui ont eu lieu avec le coup d'État de mars 2012, la guerre dans le nord et l'intervention française posent de multiples questions sur ce pays, mais aussi sur l'ensemble de l'Afrique sahélo-soudanienne, voire de l'Afrique tout entière. Ici, Hawa Coulibaly et Stéphanie Lima s'interrogent sur la crise de l'État et celle des territoires qui composent le pays. Elles montrent que pour comprendre la situation actuelle, il faut mener une réflexion d'ensemble sur l'histoire du Mali, sur la diversité des sociétés locales, sur les limites d'une démocratie poussée par l'étranger et les institutions internationales, sur les problèmes posés par une décentralisation inachevée. Mais la discussion n'est pas close...

7 La rubrique Sur le métier est consacrée au parcours d'Antoine Haumont, géographe qui a marqué la discipline durant plusieurs décennies et dont l'itinéraire, des années 1950 au début des années 2000, est à certains égards exemplaire. En effet, du texte d'Alexis Sierra, élaboré à la suite de plusieurs entretiens, ressortent des questions qui vont audelà du seul itinéraire d'un géographe et amènent à réfléchir sur le positionnement de la discipline dans le champ des savoirs et les relations entre milieu scientifique, décideurs et acteurs de l'aménagement. C'est d'abord la question du rôle de la géographie dans le dialogue interdisciplinaire qui est posée, construite dans le cas d'Antoine Haumont, à partir des années 1960, autour des liens de la géographie avec la sociologie, puis plus tard avec les sciences de l'ingénieur, l'architecture et d'autres disciplines. Cette interdisciplinarité débouche sur des relations avec certains milieux professionnels, mettant en évidence la contribution des géographes dans le domaine de l'action publique. Au-delà, le parcours d'Antoine Haumont s'inscrit dans des époques 
marquées par la décolonisation, mai 1968, etc. qui rendent compte de son intérêt pour le Tiers-monde à la charnière des années 1950-1960, pour les nouvelles façons d'aborder la question de l'habitat à partir des décennies 1960-1970. À cet égard, il confirme le poids du contexte social et politique dans la construction des savoirs. 